\title{
2 Definition und Neuordnung der Erstattungsfähigkeit
}

\subsection{Definition Medizinprodukte}

MP sind gekennzeichnet durch eine immense Heterogenität. Sie erstrecken sich über ein Spektrum, das von Pflastern und Verbandsmitteln über Kanülen bis hin zu technischen medizinischen Großgeräten, wie Magnet-ResonanzTomographen, Herzschrittmachern und Implantaten reicht. ${ }^{[4]}$ Die Anwendungsbereiche gehen von der Therapie und Diagnostik bis zur Prävention und Rehabilitation. Schätzungen gehen von 10.000 Produktgruppen sowie von einer Gesamtzahl von 400.0oo verschiedenen MP aus. ${ }^{[31]}$

Per Definition erfüllen MP einen medizinischen Zweck und sind für die Anwendung am Menschen bestimmt. ${ }^{[32]}$ Gemäß $\mathbb{3}$ Medizinproduktegesetz (MPG) sind MP ,alle einzeln oder miteinander verbunden verwendeten Instrumente, Apparate, Vorrichtungen, Software, Stoffe und Zubereitungen aus Stoffen oder andere Gegenstände einschließlich der vom Hersteller speziell zur Anwendung für diagnostische oder therapeutische Zwecke bestimmten und für ein einwandfreies Funktionieren des MPs eingesetzten Software“. MP müssen vom Hersteller zur Anwendung für Menschen mittels ihrer Funktionen für diagnostische oder therapeutische Zwecke bestimmt sein.

Grundsätzlich lässt sich festhalten, dass alle zu medizinischen Zwecken im oder am menschlichen Körper eingesetzten Produkte, die überwiegend auf physikalischem Weg funktionieren, MP sind.[4] Ihre Zweckbestimmung kön- 
nen MP im Allgemeinen auf mechanische, chemische, physikalisch-chemische oder ähnliche Funktionsweise erreichen. Aus diesem Grund haben Hersteller von MP die Funktionstauglichkeit der Produkte nachzuweisen und nicht wie bei Arzneimitteln einen Wirksamkeitsnachweis zu erbringen. ${ }^{[4]}$

\subsection{Produktklassifizierung}

Jedes MP, das in Deutschland in den Verkehr gebracht oder in Betrieb genommen wird, muss die „Grundlegenden Anforderungen“ erfüllen. ${ }^{[33]}$ Aus diesem Grund ist jeder Hersteller verpflichtet, das MP entsprechend seiner Zweckbestimmung und unter Berücksichtigung von Definitionen und Anwendungsregeln nach vorgegebenen Direktiven zu klassifizieren ${ }^{[33]}$, d.h. einer Klasse zuzuweisen. Die Klassifizierung obliegt der Eigenverantwortung des Herstellers anhand der Vorgaben der Richtlinie 98/79/EG 189 der Europäischen Union und des $\$ 13$ MPG.

Europäische Richtlinien ${ }^{[34-36]}$, die in Deutschland durch das Medizinproduktegesetz $^{[3]}$ sowie weiteren Verordnungen ${ }^{[3]}$ umgesetzt werden, sehen einen nicht staatlichen Prozess für MP vor, bei dem im Rahmen eines Konformitätsbewertungsverfahrens darüber entschieden wird, ob das Produkt grundlegende Anforderungen an Sicherheit und Leistungsfähigkeit erfüllt. Die CE-Kennzeichnung des MP, nach erfolgreichem Abschluss der Bewertung, erlaubt die Inbetriebnahme sowie Handelsfreiheit im europäischen Wirtschaftraum. [34]

Der Detaillierungsgrad des Verfahrens wird dabei durch die Zugehörigkeit des MP zu einer der vier Klassen bestimmt (s. Tab. 1). $[5,34,38,39]$

- Zur Klasse I gehören Produkte mit geringem Risikopotenzial, wie Kompressionsstrümpfe, Gehhilfen, orthopädische Hilfsmittel, Tupfer und Krankenpflegeartikel. Diese MP sind grundsätzlich nicht invasiv.

- MP der Klasse IIa sind solche mit mittlerem Risikopotenzial, z.B. Spritzen, Atembeutel, die meisten Katheter und Hörgeräte.

- In Klasse IIb fallen Produkte, die ein erhöhtes Risikopotenzial aufweisen, wie chirurgisch invasive Einmalprodukte, Kompressen zur Behandlung von Brandwunden, Blutwärmegeräte und Produkte zur Empfängnisverhütung.

- Die Klasse III enthält Produkte mit hohem Risikopotenzial, wie künstliche Herzklappen und Herzschrittmacher, resorbierbare Materialien und Produkte mit Arzneimittelbestandteilen. $[4,5,39]$

Die Zuordnung zu den unterschiedlichen Klassen basiert in Europa sowohl auf praktischen als auch ökonomischen Erwägungen, weil die Zertifizierungskosten und der Aufwand bei Medizinprodukten mit geringerem Gefährdungspotenzial geringer ausfallen. ${ }^{[4]}$ Die Klassifizierung richtet sich nach der potenziellen Gefährlichkeit des MP und berücksichtigt die möglichen Risiken in Zusammenhang mit der technischen Auslegung der Produkte und ihrer Herstellung. ${ }^{[4]}$ 
Tab. 1 Klassifizierung von Medizinprodukten

\begin{tabular}{|c|c|c|c|}
\hline Klasse & $\begin{array}{l}\text { Risiko- } \\
\text { potenzial }\end{array}$ & $\begin{array}{l}\text { Einbindung } \\
\text { „Benannter Stellen“ } \\
\text { in den Zertifizierungs- } \\
\text { prozess }\end{array}$ & Beispiele \\
\hline Klasse I & Niedrig & Keine & $\begin{array}{l}\text { Brillen, Stethoskope, Mundspatel, Urinflaschen, } \\
\text { Halskrausen, Inkontinenzeinlagen, Dekubitus- } \\
\text { matratzen }\end{array}$ \\
\hline Klasse Ila & Mittel & Produktherstellung & $\begin{array}{l}\text { Externe Hörgeräte, Kontaktlinsen, Blasenkatheter, } \\
\text { Kanülen, diagnostischer Ultraschall }\end{array}$ \\
\hline Klasse IIb & Erhöht & $\begin{array}{l}\text { Produktherstellung } \\
\text { und -auslegung }\end{array}$ & $\begin{array}{l}\text { Stents, Dialysatoren, chirurgische Laser, externe } \\
\text { Herzschrittmacher, Defibrillatoren, Röntgengeräte, } \\
\text { Kondome }\end{array}$ \\
\hline Klasse III & Hoch & $\begin{array}{l}\text { Produktherstellung } \\
\text { und -auslegung }\end{array}$ & $\begin{array}{l}\text { Herzklappen, Hüft-, Schulter- \& Kniegelenk- } \\
\text { prothesen, Brustimplantate, intrakranielle Stents, } \\
\text { koronare Stents }\end{array}$ \\
\hline
\end{tabular}

\subsection{Konformitätsbewertungsverfahren und Erprobungsregelung}

\section{CE-Kennzeichnung und Grundlegende Anforderungen}

Gemäß $\mathbb{6}$ Abs. 1 MPG darf ein MP bis auf einige dort genannte Ausnahmen nur in Verkehr gebracht werden, wenn es eine CE-Kennzeichnung besitzt. Das CE-Kennzeichen ist eine EU-Kennzeichnung und stellt die Übereinstimmung mit den europäischen Normen sowie die Einhaltung der nationalen Medizinproduktegesetze dar, welche jedoch auf fünf Jahre begrenzt ist. Das CE-Zeichen bestätigt im Europäischen Wirtschaftsraum die „Konformität“ und „Verkehrsfähigkeit“ eines MP. Das CE-Zeichen gilt als Nachweis, dass eine Risikoanalyse sowie klinische Bewertung stattgefunden haben. ${ }^{[40,41]}$

Mit der CE-Kennzeichnung wird die Zulässigkeit von Herstellung und Betrieb im europäischen Wirtschaftsraum erklärt. ${ }^{[4]}$ Damit kann das MP ungehindert in den Verkehr gebracht und vertrieben werden. ${ }^{[42]}$ Zudem gilt eine CE-Kennzeichnung als Grundvoraussetzung für eine Kostenerstattung von MP im Rahmen der GKV.

Um eine CE-Kennung für ein MP der Klasse IIa oder höher zu erhalten, stellt der Hersteller einen Antrag an eine sogenannte Benannte Stelle, von denen in Deutschland derzeit 16 durch das BMG beziehungsweise durch die Zentralstelle der Länder für Gesundheitsschutz bei Arzneimitteln und MP akkreditiert sind. Hersteller können innerhalb der EU frei entscheiden, von welcher Benannten Stelle sie ein Produkt zertifizieren lassen wollen. [34]

Die Grundlegenden Anforderungen fokussieren den Nachweis der Sicherheit eines CE-gekennzeichneten MP, es muss darüber hinaus funktionstauglich 
und leistungsfähig sein. ${ }^{[4]}$ Die Bewertung und Feststellung der Übereinstimmung der MP mit den Grundlegenden Anforderungen nach $₫ 7$ MPG regelt die Verordnung über MP (MPV).

Bestandteil der Grundlegenden Anforderungen gem. $\mathbb{} 7$ MPG ist die klinische Bewertung, die für jedes Produkt durchzuführen ist. $\$ 19 \mathrm{MPG}$ fordert den Beleg für die Erfüllung des vorgesehenen medizinischen Verwendungszwecks durch eine klinische Bewertung. Im Rahmen der klinischen Bewertung ist auch die Unbedenklichkeit der MP durch Abwägung von Risiken und Nutzen zu belegen. Der Umfang der klinischen Bewertung ist aufgrund der unterschiedlichen Komplexität und des variierenden Risikopotenzials sehr unterschiedlich. Die klinische Bewertung kann einerseits auf bereits vorhandene wissenschaftliche Erkenntnisse gestützt werden und ist zusätzlich durch einen Bericht mit kritischer Würdigung zu ergänzen. ${ }^{[33]}$ Neben diesem Literaturweg kommt auch eine klinische Prüfung in Betracht. Sie stellt einen Unterfall zur klinischen Bewertung dar und ist nicht etwa mit dieser gleichzusetzen. Sie ist ein möglicher Weg, die Anforderungen an die klinische Bewertung zu erfüllen. ${ }^{[4]}$

Die Entscheidung, ob eine klinische Bewertung oder eine klinische Prüfung durchgeführt wird, trifft der Hersteller. Ist die Einschaltung einer Benannten Stelle erforderlich, so hat diese über die Notwendigkeit einer klinischen Prüfung zu entscheiden. Durch die klinische Bewertung oder die klinische Prüfung wird die medizinische Leistungsfähigkeit der Produkte im Hinblick auf die Zweckbestimmung nachgewiesen. ${ }^{[4]}$

\section{Konformitätsbewertung}

Die CE-Kennzeichnung darf nur erfolgen, wenn die Grundlegenden Anforderungen eingehalten werden und wenn der Hersteller ein Konformitätsbewertungsverfahren durchführt hat. Die Konformitätsbewertung soll objektive Hinweise auf Sicherheit, Leistung, Nutzen und Risiken geben. ${ }^{[43,}{ }^{44]}$ Ziel des Konformitätsbewertungsverfahrens ist es, die Behörden in die Lage zu versetzen, sich zu vergewissern, dass die in Verkehr gebrachten Produkte insbesondere in Bezug auf den Gesundheitsschutz und die Sicherheit der Benutzer und Verbraucher den Anforderungen der Richtlinien gerecht werden. ${ }^{[4,33]}$ Welches gesetzlich festgelegte Konformitätsbewertungsverfahren ein bestimmtes Produkt durchlaufen muss, legt die Zuordnung zu einer der vier Klassen fest. Mit einer erhöhten Gefährlichkeit eines Produktes steigt das Ausmaß der Kontrolle im Rahmen eines durchzuführenden (modularen) Konformitätsbewertungsverfahrens. ${ }^{[4]}$

\section{Erlaubnis- und Verbotsvorbehalt}

Die Mehrzahl gerade der risikobehafteten MP (Klasse IIb und III) wird im stationären Sektor eingesetzt. Während NUB im ambulanten Sektor nur dann 
angewendet und erstattet werden dürfen, wenn der G-BA einen entsprechenden positiven Beschluss gefasst hat (Verbot mit Erlaubnisvorbehalt), können im stationären Bereich alle Leistungen erbracht werden, sofern der G-BA die Erstattungsfähigkeit nicht untersagt (Erlaubnis mit Verbotsvorbehalt). ${ }^{[9]}$ Laut $\mathbb{} 137 \mathrm{C}$ Abs. 1 SGB V können NUB in der stationären Versorgung grundsätzlich ohne vorherige Prüfung durch den G-BA zu Lasten der GKV erbracht werden. Dies gilt sowohl für NUB mit als auch ohne Anwendung von MP. Zudem können Untersuchungs- und Behandlungsmethoden, zu denen der G-BA noch keine Entscheidung getroffen hat, laut $\$ 137 \mathrm{C}$ Abs. 3 SGB V im Rahmen einer Krankenhausbehandlung angewandt werden, wenn sie das Potenzial einer erforderlichen Behandlungsalternative bieten und ihre Anwendung nach den Regeln der ärztlichen Kunst erfolgt, sie also insbesondere medizinisch indiziert und notwendig sind. Der Ablauf des Verfahrens zur Bewertung neuer Untersuchungs-

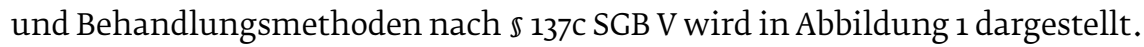

\section{Erprobungsregelung}

Durch das Versorgungsstrukturgesetz wurden 2012 die Regelungen zur Methodenbewertung ambulanter und stationärer nicht-medikamentöser Verfahren vom G-BA erneuert. Für Untersuchungs- und Behandlungsmethoden nach $\$ 135$ SGB V (ambulant) oder $\$ 137 \mathrm{C}$ SGB V (stationär), bei denen der G-BA im Rahmen der Methodenbewertung zu der Feststellung gelangt, dass der Nutzen noch nicht hinreichend belegt ist, sich aber ein Potenzial zur erforderlichen Behandlungsalternative erkennen lässt, hat der Ausschuss die Möglichkeit eine Richtlinie zur Erprobung nach $\mathbb{\$} 137$ e SGB V zu beschließen.

Der G-BA muss die Untersuchungs- und Behandlungsmethode von der Erstattung der Gesetzlichen Krankenversicherung ausschließen, sobald nach der abgeschlossenen Erprobung festgestellt wird, dass die Methode im Rahmen einer Krankenhausbehandlung nicht den Kriterien gemäß \$137c Abs. 1 SGB V entspricht. Beruht die technische Anwendung der innovativen Methode maßgeblich auf dem Einsatz eines MP, darf der G-BA gemäß $\mathbb{1} 137 \mathrm{e}$ Abs. 6 SGB V einen Beschluss zur Erprobung nach Absatz 1 nur dann erlassen, wenn sich die Hersteller dieses MP dazu bereit erklären, die Übernahme der entstehenden Kosten für die wissenschaftliche Begleitung und Auswertung einer unabhängigen wissenschaftlichen Institution nach Absatz 5 zu gewährleisten. Für den Fall, dass eine Erprobung daran scheitert, dass eine angemessene Kostenübernahme von Herstellern oder sonstigen Unternehmen nicht zustande kommt, sieht $\mathbb{\$} 137$ e Abs. 5/6 SGB V vor, dass die Methode ausgeschlossen werden kann, da ihr Nutzen nicht hinreichend belegt ist. 
2 Definition und Neuordnung der Erstattungsfähigkeit

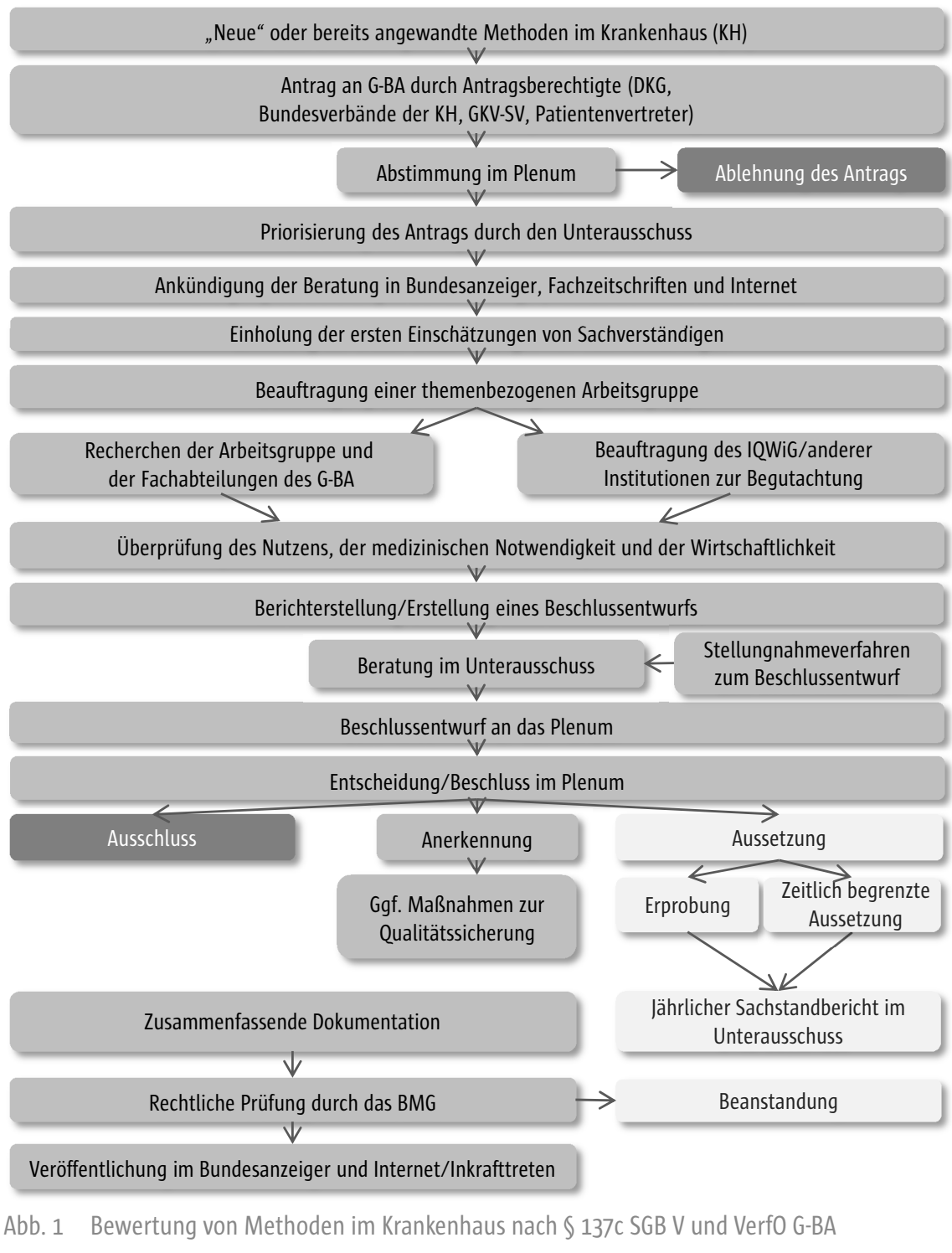

\subsection{Neuordnung der Nutzenbewertung}

Vom Bundeskabinett wurde im Dezember 2014 der Entwurf eines „Gesetzes zur Stärkung der Versorgung in der gesetzlichen Krankenversicherung“ vorgelegt. Dieser Entwurf wurde am 11. Juni 2015 durch den Bundestag beschlossen. Das Gesetz zielt darauf ab „die Nutzenbewertung neuer Methoden mit Medizinprodukten hoher Klasse zu stärken. “" ${ }^{[45]}$ Hierbei wird für neue Methoden, bei denen MP mit hoher Klasse verwendet werden und für welche die Krankenhäuser nach dem Krankenhausentgeltgesetz (\$ 6 Abs. 2 Satz 3 
KHEntgG) eine Vergütung bzw. ein Entgelt anstreben „ein systematisches, obligatorisches und fristgebundenes Verfahren zur Bewertung der Methode durch den Gemeinsamen Bundesausschuss vorgesehen." ${ }^{45]}$ Dieses verpflichtende Verfahren nach $\$ 137 \mathrm{~h}$ SGB V wird (auch) als frühe Nutzenbewertung bezeichnet. ${ }^{[46]}$ Die Bezeichnung der frühen Nutzenbewertung an dieser Stelle sollte jedoch nicht mit dem Arzneimittelmarkt-Neuordnungsgesetz (AMNOG) gleichgesetzt werden. ${ }^{[47]}$ Innerhalb des $\mathbb{1} 137 \mathrm{~h}$ SGB V werden der Inhalt, der grobe Ablauf, die Zuständigkeit, die Beteiligung verschiedener Institutionen und die Anwendbarkeit dieses Nutzenbewertungsverfahrens definiert.

Das Verfahren zur Nutzenbewertung beginnt regulär mit zwei parallel ablaufenden Prozessen. Zum einen stellt ein Krankenhaus, das eine NUB erbringen möchte, die maßgeblich auf der Anwendung eines MP hoher Klasse mit besonders invasivem Charakter beruht, erstmalig zu der jeweiligen Methode eine Anfrage nach $\$ 6$ Abs. 2 Satz 3 des Krankenhausentgeltgesetzes (Antrag auf Vergütung einer NUB beim InEK). Zugleich übermittelt das antragstellende Krankenhaus im Benehmen mit dem Hersteller Informationen über die NUB mit neuem theoretisch-wissenschaftlichem Konzept dem G-BA. Vor dem regelmäßigen Beginn des Verfahrens soll sich das Krankenhaus bereits einen Überblick über Wirksamkeit und Nutzen der Methode verschaffen und hierzu Informationen vom Hersteller, der das anzuwendende MP produziert, einholen. [45]

Nach dem Eingang der Informationen beim G-BA, prüft dieser innerhalb von zwei Wochen, ob es sich um eine Methode handelt, die auf einem neuen theoretisch-wissenschaftlichen Konzept und maßgeblich auf dem Einsatz eines MP mit hoher Klasse und besonders invasivem Charakter beruht. ${ }^{[45]}$ Kommt der G-BA zu einem positiven Ergebnis, wird die Beratung zu dieser Methode innerhalb von zwei Wochen im Internet öffentlich bekanntgemacht. Mit dieser Bekanntmachung geht ein Aufruf an alle Krankenhäuser, die eine Erbringung der neuen Untersuchungs- oder Behandlungsmethode vorsehen, sowie die jeweils betroffenen Medizinproduktehersteller einher, weitere wichtige Informationen zur betreffenden Methode innerhalb eines Monats an den G-BA zu übermitteln. ${ }^{[45]}$

Liegen dem G-BA alle relevanten Unterlagen vor, beginnt die dreimonatige Frist des Bewertungsverfahrens. Im Rahmen des Bewertungsprozesses kann der G-BA das Institut für Qualität und Wirtschaftlichkeit im Gesundheitswesen mit der Bewertung der vorliegenden Unterlagen beauftragen. [45] Das Bewertungsverfahren lässt drei mögliche abschließende Beschlussvarianten zu (s. Abb. 2):

1. ein belegbarer ausreichender Nutzen der NUB liegt vor $\rightarrow$ Anerkennung

2. Nutzen ist noch nicht hinreichend belegt, aber die Methode bietet das Potenzial einer erforderlichen Behandlungsalternative $\rightarrow$ Anerkennung eines potenziellen Nutzens/Potenzials und ggf. Erprobung

3. NUB ist schädlich oder unwirksam und bietet kein Potenzialals Behandlungsalternative $\rightarrow$ Ausschluss 


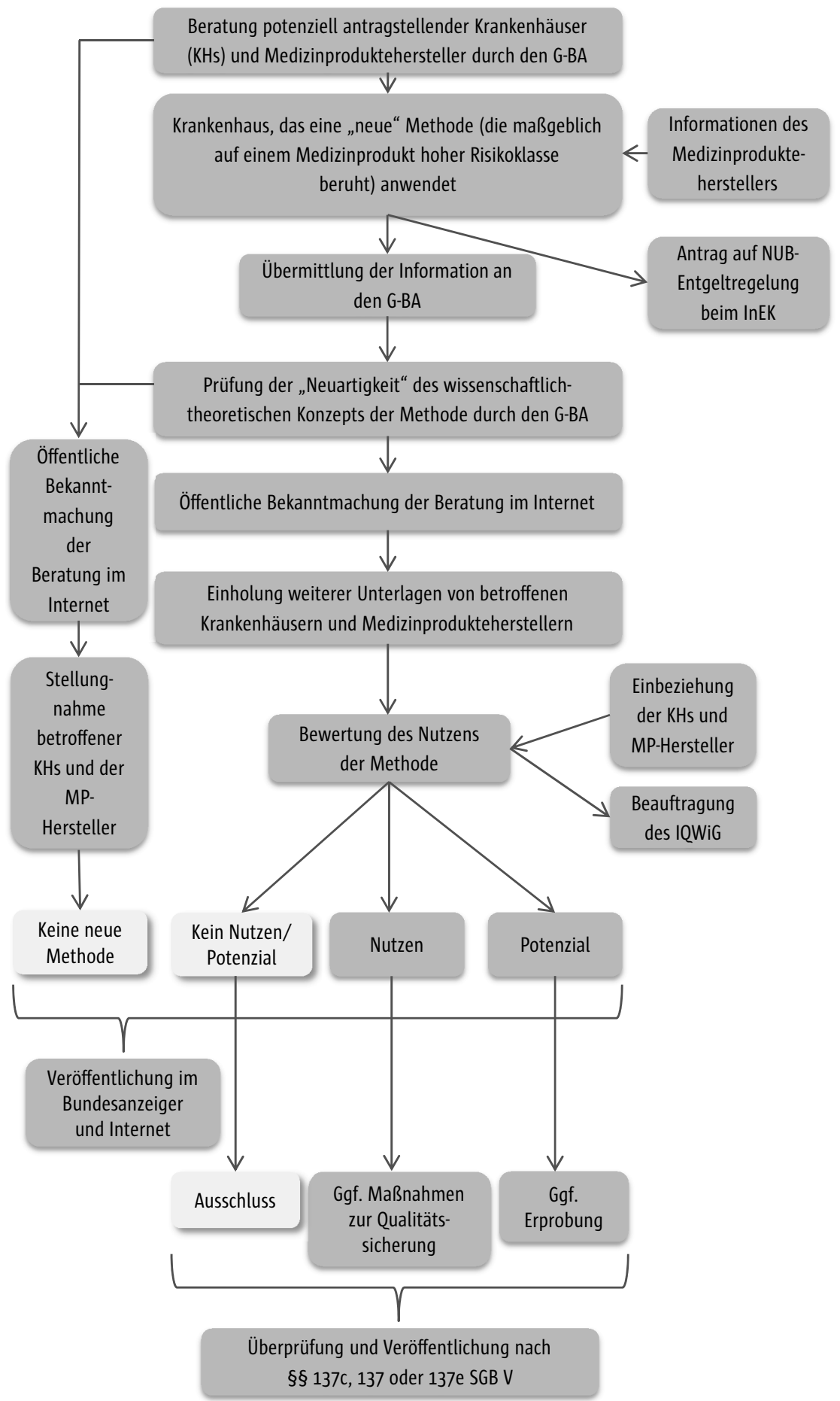

Abb. 2 Nutzenbewertung von NUB im Krankenhaus nach $\$ 137 \mathrm{~h} \mathrm{SGB} \mathrm{V}$ 
Die Anerkennung des Nutzens einer NUB, deren Anwendung maßgeblich auf einem MP mit hoher Klasse und besonders invasivem Charakter beruht, ermöglicht die Vereinbarung einer NUB-Vergütung durch einen Antrag nach $\$ 6$ Absatz 2 Satz 1 des KHEntG, insofern die Erbringung der Methode nicht sachgerecht mit bereits bestehenden pauschalierten Sätzen vergütet werden kann ( $\$ 137$ h, Abs. 3 SGB V). Darüber hinaus überprüft der G-BA, ob die Erbringung der Methode spezifische Anforderungen an die Qualität der Leistungserbringung erfordert und regelt diese gegebenenfalls in der dafür vorgesehenen Richtlinie. ${ }^{[45]}$

Können die eingereichten Unterlagen den Nutzen zwar nicht ausreichend belegen, lassen aber ein Potenzial diesbezüglich erkennen, beschließt der G-BA innerhalb von sechs Monaten über eine Richtlinie zur Erprobung. Im Falle einer Erprobung wird die Methode weiteren Studien unterzogen, um den potenziellen Nutzen zu belegen. Die Erprobung von Untersuchungs- und Behandlungsmethoden ist im $\mathbb{s} 137 \mathrm{e}$ SGB V geregelt.

Wenn eine Methode als unwirksam oder schädlich eingestuft wird und somit keine potenziell erforderliche Behandlungsalternative darstellt, ist eine Vereinbarung nach $\$ 6$ Absatz 2 Satz 1 des Krankenhausentgeltgesetzes oder nach \$ 6 Abs. 2 Satz 1 der Bundespflegesatzverordnung ausgeschlossen. Der G-BA entscheidet unverzüglich über eine Richtlinie nach $\$ 137$ C SGB V und nimmt die Methode in die Liste der ausgeschlossenen Methoden in der Richtlinie Methoden Krankenhausbehandlung nach $\$ 137$ c SGB V auf. Die Erstattung solcher ausgeschlossener Methoden durch die GKV ist folglich untersagt. [45]

Vor dem gesamten Verfahren haben Krankenhäuser und auch Hersteller von MP die Möglichkeit, eine Beratung durch den G-BA kostenfrei in Anspruch zu nehmen. Im Rahmen dieser Beratung erhalten das potenziell antragstellende Krankenhaus und/oder der Hersteller Informationen über die Voraussetzungen und Anforderungen eines Antrags im Hinblick auf konkrete Methoden und schon vorab eine Feststellung der Neuartigkeit des theoretisch-wissenschaftlichen Konzepts der Methode. Die Beratung ist jedoch kein verpflichtender Abschnitt des Verfahrensablaufs, sie ist ein freiwilliges Angebot und dient der Entzerrung des Gesamtprozesses. ${ }^{[45]}$

Wird innerhalb dieser optionalen Beratung oder in der Prüfung der Neuartigkeit des Konzepts der Methode durch den G-BA festgestellt, dass es sich um eine bekannte Methode handelt, die bereits angewandt wird, trifft der G-BA diesbezüglich einen Beschluss. Vor dieser Beschlussfassung wird diese Absicht im Internet bekannt gegeben und anderen Krankenhäusern und Medizinprodukteherstellern die Möglichkeit gegeben, weitere relevante Unterlagen zur Neuartigkeit des Konzepts an den G-BA zu übermitteln. Außerdem werden Stellungnahmen von den betroffenen Krankenhäusern und Medizinprodukteherstellern eingeholt und in den Entscheidungsprozess einbezogen. ${ }^{[45]}$ 
Den Abschluss des Verfahrens bildet die Veröffentlichung des Beschlusses und der Tragenden Gründe im Bundesanzeiger und dem Internet. Die rechtliche Prüfung des Beschlusses durch das BMG nach $₫ 94$ Abs. 1 SGB V ist nicht vorgesehen, da es sich nicht um einen Richtlinienbeschluss handelt. ${ }^{[45]}$ Jedoch wird die daraus resultierende Richtlinienänderung vom BMG überprüft.

\subsection{Neue Methode/Neues theoretisch-wissenschaftliches Konzept}

Die Begrifflichkeiten „neue Methode“, „neues theoretisch-wissenschaftliches Konzept" oder NUB sind im Kontext der Nutzenbewertung von MP noch nicht vollumfänglich definiert. Jedoch bestehen bereits in anderen Zusammenhängen Definitionen oder anfängliche Erklärungen.

Der Gesetzentwurf des GKV-VSG selbst liefert eine erste Beschreibung des Ausdrucks. Demzufolge erfüllt eine NUB den Begriff „,neuartiges theoretischwissenschaftliches Konzept “ dann, wenn sich entweder das Wirkprinzip oder das Anwendungsgebiet der NUB von anderen Methoden, die in der stationären Versorgung bereits angewandt werden, in der systematischen Herangehensweise wesentlich unterscheidet. ${ }^{[45]}$ Die genaue Definition des Begriffs wird durch eine Rechtsverordnung zum 31. Dezember 2015 von den Bundesministerien für Gesundheit sowie Bildung und Forschung festgelegt.[45]

In der Verfahrensordnung des G-BA findet sich im zweiten Kapitel in $\mathbb{2} 2$ eine Definition des Terminus „neue Methode“. Diese Definition steht im Zusammenhang mit der Bewertung von Methoden nach $\mathbb{} 135$ SGB V, also für neue Methoden im ambulanten vertragsärztlichen Sektor. Der Gemeinsame Bundesausschuss definiert den Ausdruck „neue Methode“ als Leistungen, die

1. im bisherigen Abrechnungssystem nicht abgebildet sind oder

2. zwar im Abrechnungssystem abgebildet sind, aber für eine neue Indikation oder mittels neuer Erbringungsart erfolgen oder

3. im Abrechnungssystem abgebildet sind, aber eine wesentliche Änderung bezüglich der Indikation oder der Erbringungsart vorgenommen wurde.

Kommt es zu keiner einheitlichen Lösung oder Unstimmigkeiten, wird eine Stellungnahme des Bewertungsausschusses (nach $\mathbb{8} 87$ SGB V) eingeholt. ${ }^{[48]}$ Neben der Verfahrensordnung stellt der G-BA eine weitere Definition zur Verfügung. Diese stützt sich auf das Urteil des Bundessozialgerichts in Kassel von 1998: „Nach ständiger Rechtsprechung ist eine Methode eine medizinische Vorgehensweise, der ein eigenes theoretisch-wissenschaftliches Konzept zugrunde liegt, welches sie von anderen Verfahren unterscheidet und das ihre systematische Anwendung in der Untersuchung und Behandlung bestimmter Krankheiten rechtfertigen soll (nach BSG, Urt. v. 23.07.1998, Az.: B 1 KR 19/96 R). “[49] 


\subsection{Nutzenbewertung und Health Technology Assessment}

Auch im Kontext der Nutzenbewertung operiert der Gesetzgeber mit unscharfen methodischen Begriffen. Die Vorgaben des Gesetzgebers stützen sich nicht auf allgemein akzeptierte Definitionen und es fehlt ein etabliertes System von Nutzeneinheiten oder Nutzwerten. Die betrachteten Konzepte und Konstrukte wie Gesundheit, klinischer Nutzen oder Patientennutzen, sind in aller Regel komplex und nicht direkt messbar. Die direkte Messung von Gesundheit und dem hieraus resultierenden Patientennutzen sind dagegen nicht möglich.

Allgemeingefasst meint der Begriff „Nutzen“ aber eine positive Auswirkung und wird in der Medizintechnik den Begrifflichkeiten Risiko und Schaden gegenübergestellt. ${ }^{[50]}$ Bezogen auf die Nutzenbewertung ist hierbei auch die Kausalität wichtigg[5] ${ }^{[5 . h}$. „die kausale Verbindung von Intervention und Auswirkung“" 25$]$. Anders als im Bereich der Arzneimittel besteht in der Medizintechnik noch keine klare und im Gesetz festgeschriebene Definition der Kriterien zur Nutzenbewertung von MP. ${ }^{[5,52]}$ Eine Behandlungsstrategie mit einem MP hat demnach dann einen Nutzen, wenn sie positive Auswirkungen auf das Behandlungsergebnis sowie die längerfristige Lebensqualität des Patienten hat und dabei möglichst wenig Komplikationen (Mortalität, Morbidität) verursacht. Diener et al. ${ }^{[19]}$ verweisen in diesem Zusammenhang jedoch darauf, dass neben der sorgfältigen Abwägung des inhärenten Risikopotenzials und der Vorteile für den Patienten für eine umfassende Einschätzung des Nutzens auch klinisch-ökonomische Analysen berücksichtigt werden müssen. ${ }^{[19]}$

Um zu einer effizienten Ressourcenallokation nach dem Inverkehrbringen von MP zu gelangen, wurden in vielen Ländern formale Verfahren des Health Technology Assessments (HTA) etabliert. ${ }^{[17]}$ HTA-Verfahren beinhalten die Identifizierung und kritische Beurteilung der vorhandenen Evidenzgrundlage, um die klinische und ökonomische Bewertung konkurrierender Gesundheitstechnologien zu beeinflussen. ${ }^{[53]}$ Luce et al. definieren HTA als „eine Methode der Evidenzsynthese, welche die Evidenz bezüglich der klinischen Wirksamkeit, Sicherheit, Wirtschaftlichkeit berücksichtigt (...)HTAs werden hauptsächlich zur Beeinflussung von Erstattungsentscheidungen eingesetzt, wobei HTAs in diesem Fall Nutzen-Schaden-Bewertungen und ökonomische Bewertung enthalten sollten“. ${ }^{[54]}$

Das HTA, das einen interdisziplinären und -professionellen Ansatz verfolgt, baut auf dem Kerngedanken der evidenzbasierten Medizin auf. ${ }^{[55,56]}$ Anders als in der EbM, in der primär wissenschaftliche, klinische Studien als Entscheidungsgrundlage herangezogen werden, ist der Betrachtungshorizont im HTA deutlich breiter. ${ }^{[57]}$ Adressat des HTA sind zumeist Politik und Kostenträger. HTA kann als „Methode der systematischen, interdisziplinären Synthese sowie der transparenten und politikrelevanten Aufbereitung von Wissen zu (neuen, innovativen, aber auch etablierten) medizinischen Interventionen "[58] ver- 
standen werden. Anders als in der EbM wird in der Bewertung im Rahmen eines HTA jedoch nicht nur die klinische Wirksamkeit einer Technologie berücksichtigt, sondern es fließen auch ökonomische, soziale, juristische und ethische Aspekte in die Betrachtung mit ein (s. Abb. 3).[5] Diese Herangehensweise macht das HTA zu einem aussagekräftigen und unterstützenden Instrument in der politischen Entscheidungsfindung und der Verteilung begrenzter Ressourcen. $\left.{ }^{[57}, 58\right]$

Das „HTA-Core-Modell“ - ein „HTA-Modell für die schnelle und frühe Beurteilung (Rapid Relative Effectiveness Assessment, REA) der relativen Wirksamkeit von Arzneimitteln“ - wurde von Experten von 18 europäischen HTA-Institutionen entwickelt. Das Modell stellt einen Konsens der unverbindlichen Empfehlungen der EUnetHTA-Mitglieder dar. Das HTA-Core-Modell definiert die Elemente, die in einem HTA in Betracht gezogen werden sollten und erleichtert die standardisierte Berichterstattung. Die Ziele sind Informationen zu teilen, um Doppelarbeit nationaler HTA-Organisationen zu vermeiden und die Anpassung der Informationen an nationale HTA-Berichte zu ermöglichen sowie die Kooperation in der Erstellung von HTA-Berichten (durch mehrere Agenturen) zu erleichtern. Insgesamt wurden durch das Netzwerk neun Richtlinien entwickelt, anhand derer die Gutachter (HTA-Agenturen) die Evidenz beurteilen und die Daten aufarbeiten sollen, die als Teil eines REA präsentiert werden. Diese neun Richtlinien umfassen folgende Punkte:

1. Klinische Endpunkte

2. Kombi-Endpunkte

3. Surrogatendpunkte

4. Sicherheit

5. Gesundheitsbezogene Lebensqualität

6. Kriterien für die Auswahl der am besten geeigneten Komparator(en)

7. Direkte und indirekte Vergleiche

8. Interne Validität

9. Anwendbarkeit der Evidenz im Kontext der Beurteilung der relativen Wirksamkeit. ${ }^{[60]}$

\subsection{Phasen der Nutzenbewertung}

Zielsetzung einer Intervention ist es, Patientennutzen zu schaffen. Zielsetzung der Nutzenbewertung hingegen ist die Operationalisierung des Patientennutzens, um eine rationale Wahlentscheidung über Interventionen zu ermöglichen. Das Verfahren der Nutzenbewertung sollte eine nachvollziehbare Bewertung durch eine transparente Arbeitsweise garantieren. Die dem Verfahren zugrundeliegenden wissenschaftlichen Grundlagen sollten dokumentiert werden und darüber hinaus sollte sich das Verfahren an den gesetzlichen Grundlagen orientieren. 


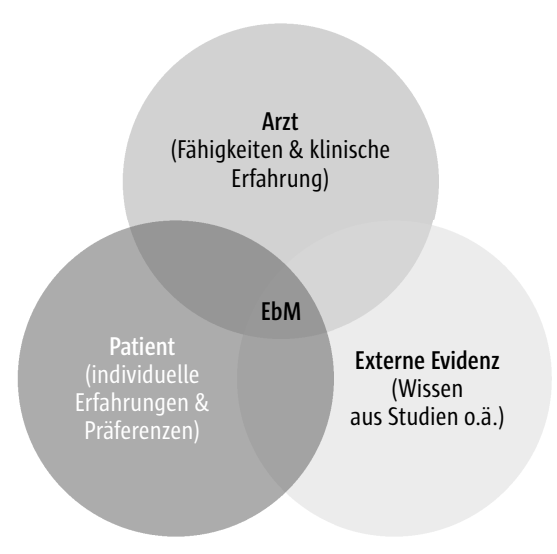

EbM

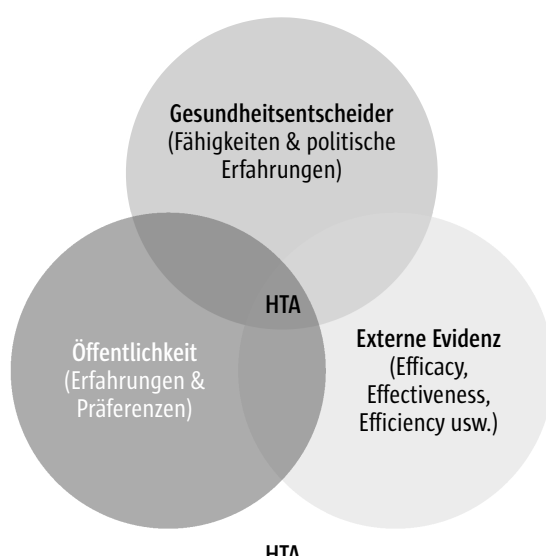

HTA

Abb. 3 HTA versus EbM

Crundsätzlich kann die Nutzenbewertung in drei Phasen unterteilt werden:

Das Messen von kausalen Effekten einer Intervention, wobei klinische und nicht-klinische Zielgrößen beim Patienten, einer Patientenpopulation oder den Bürgern mit einem dafür geeigneten Studiendesign gemessen werden. Endpunkte sollten zuverlässig und korrekt Änderungen des Gesundheitszustandes abbilden. In den meisten Fällen werden neben dem primären Endpunkt auch sekundäre Endpunkte bei der Messung berücksichtigt. Damit kann von einem multikriteriellen Entscheidungsproblem ausgegangen werden. Unter anderem sind hier die folgenden Arbeitsschritte relevant: Definition der Zielkriterien, Festlegung der Messgrößen, Zuordnung der Effekte im Experiment, Bewertung der Unsicherheit und Interpretation der Ergebnisse bzw. Ergebnissicherheit.

Die Bewertung der multiplen gemessenen Effekte erfolgt im nächsten Schritt. Kausal begründete Effekte aus Phase 1 können einen Nutzen oder Schaden für den Patienten darstellen. Nutzen im eigentlichen Sinne resultiert erst aus der Bewertung der klinischen Effekte und erfolgt in der Regel aus der Perspektive des Konsumenten. Das Bewerten ist Gegenstand der Ökonomie und Statistik, insbesondere der Entscheidungstheorie. Zielsetzung ist die systematische Aggregation unterschiedlicher Zielerreichungsgrade, d.h. die Zusammenfassung aller Informationen über die Effekte in einem (eindimensionalen) Nutzenmaß. Ergebnis der Bewertung ist die Bereitstellung von Informationen, d.h. indikationsspezifischen oder indikationsübergreifenden Kennzahlen, für eine Entscheidung. Der rationale Vergleich von Interventionen erfolgt anhand dieses (eindimensionalen) Nutzenmaßes, als notwendige Bedingung für die Ermittlung des Nutzens einer Intervention über die Vergleichsintervention. Folgende Aspekte können dabei im Mittelpunkt stehen: Relevanz der Zielkriterien, Werturteile im Hinblick auf die Gewichtung und Vergleichbarkeit der Effekte sowie Annahmen über die Aggregation multipler Zielkriterien. 
Die Entscheidung über die Intervention(en) wird auf Basis der Informationen über den Nutzen aus Phase 2 getroffen. Eine rational begründete Entscheidung über Interventionen basiert auf vorab definierten Zielen und vorhandenen transparenten Werturteilen/Wertmaßstäben. Die Entscheidungskriterien können von den Zielen der Effektivität und der Wirtschaftlichkeit einer Intervention abhängig sein. Die rational begründete Entscheidung orientiert sich neben den kausal begründeten Effekten (Zielerreichungsgraden) auch an den Präferenzen der Betroffenen, als Ausdruck der Wertvorstellungen (Werturteilen oder Wertmaßstäben). Die Zielsetzung der Entscheidung ist die rationale Auswahl der optimalen Handlungsalternative (oft unter Unsicherheit). Folgende Aspekte können dabei interessant sein: Konkretisierung der Entscheidungslogik, Auswahl der Wirtschaftlichkeitsprinzipien (Minimum- und Maximumprinzip), Unterstützung/Konfliktlösung derVerhandlungspartner und Berücksichtigung ethischer und moralischer Rahmenbedingungen.

Dieser umfassende Prozess über alle drei Phasen kann als Verfahren der Nutzenbewertung bezeichnet werden. Eine transparente Nutzenbewertung und Entscheidung über die Erstattungsfähigkeit, die sich konsistent am Patientennutzen und der Wirtschaftlichkeit orientiert, maximiert die Wohlfahrt für die Bevölkerung und schafft Planungssicherheit für das innovative Gesundheitsunternehmen. Es bedarf geeigneter Methoden und Instrumente, welche die Evidenz hinsichtlich der kausalen Effekte und der Präferenzen über das gesamte Bewertungsverfahren berücksichtigen. So kann einer „subjektiv geprägten und damit häufig verzerrten Bewertung " vorgebeugt werden. ${ }^{[1]}$ Dazu gehören die Messung klinischer und nicht-klinischer Effekte, die Bewertung dieser gemessenen Zielkriterien (klinische oder nicht-klinische Endpunkte) sowie die Anwendung einer transparenten Entscheidungslogik. Die dafür notwendigen Methoden und Kriterien müssen ferner den anerkannten internationalen Standards der EbM, aber auch der Gesundheitsökonomie entsprechen. In den folgenden Kapiteln des Gutachtens soll daher auf diese drei Phasen detailliert eingegangen werden und entsprechende Lösungskonzepte zur Diskussion gestellt werden. 